\title{
Multiscale Computational Analysis of the Effect on Heart Rate of a HCN4 Gene Double Mutation: From the Single Channel to the Clinical Phenotype
}

\author{
Eugenio Ricci ${ }^{1}$, Alan Fabbri ${ }^{2}$, Teun P de Boer ${ }^{2}$, Stefano Severi ${ }^{1}$ \\ ${ }^{1}$ Alma Mater Studiorum - University of Bologna, Cesena, Italy \\ ${ }^{2}$ Department of Medical Physiology, University Medical Center Utrecht, Utrecht, The Netherlands
}

\begin{abstract}
This work aims to assess: I) the effects of the I479V/A485E HCN4 channel double mutation (DM), II) the role of cellular coupling $(\rho)$ and III) the role of cellular heterogeneity $(\sigma)$ on systems of increasing complexity: ionic channel, single cell, $1 D$ fibre and $2 D$ tissue of the human SAN. The Fabbri et al. model was used to describe the human SAN cell and to build the $1 D$ and $2 D$ models, which are constituted of 100 and 2500 (50x50) cells, respectively. $\sigma=0.05,0.1,0.1873,0.3,0.4$ and $\rho=10$, $100,1000,10000$ and $\infty \mathrm{M} \Omega \cdot \mathrm{m}$ were simulated, in order to test their effect on the heart rate (HR).

The reduction of $I_{f}$ current due to the heterozygous condition $\left(g_{f}(W T 0.5+D M)=45 \%\right.$ of $\left.g_{f}(W T)\right)$ leads to an increase of the cycle length of the simulated action potential of a single cell (924 vs $814 \mathrm{~ms} ;+14 \%)$. This WT0.5+DM bradycardic effect is confirmed also by the $1 D$ model (802 vs $690 \mathrm{~ms} ;+14 \%)$ as well as by the $2 \mathrm{D}$ one (908 vs $794 \mathrm{~ms} ;+14 \%)$. These results were obtained for $\sigma=0.1873$ and $\rho=100 \mathrm{M} \Omega \cdot \mathrm{m}(50 \mathrm{M} \Omega \cdot \mathrm{m}$ in $1 D)$. Other combinations of $\sigma$ and $\rho$ can provide changes in $H R$ greater than 50\%, highlighting the importance of these two parameters in the establishment of a physiologic pacing in the human SAN.
\end{abstract}

\section{Introduction}

The sinoatrial node (SAN), is a small and complexshaped tissue, known to be the source of the electrical pulse in the heart, under physiological conditions. Its cells present indeed autorhythmicity, and have thus the ability to cyclically depolarize and trigger the propagation of the Action Potential (AP) through the entire myocardium. A central role in the onset of the AP is held by the $I_{f}$ current, which governs the diastolic depolarization phase. This current flows through the HCN4 channels, and any mutation affecting these channels can either bring to a loss or gain of function in this current [1,2]. The propagation of the AP through the cells is mediated by gap junctions. These are indeed channels through which ions can flow, thus propagating the stimulus from one cell to another. Besides the coupling, another fundamental feature of biological tissues is the cellular heterogeneity: all the cells composing the SAN have different dimensions and electrical properties. This determines a different behaviour in terms of pacing: some cells will have a higher intrinsic pacing frequency, whereas others will have a lower one. The lack of anatomical and electrophysiological data about the human SAN increases the importance of in silico simulations, which provide a valuable tool in order to promote hypotheses or test previous ones. The aims of this work are therefore those of investigating: I) the effects of the I479V/A485E HCN4 channel double mutation (DM, [3]), II) the role of cellular coupling $(\rho)$ and III) the role of cellular heterogeneity $(\sigma)$. To achieve this, the decrease in HCN4 channel conductance due to the $\mathrm{DM}$, in the heterozygous condition (WT0.5+DM) presented by the patient and relative to the WT condition, is estimated as first. We included the new value of $g_{f}$ in the single cell model and we measured the cycle length of the mutant cell. Finally, 1D and 2D models were built, and simulations were run to assess the effect of DM, $\rho$ and $\sigma$ on CL and Conduction Velocity (CV).

\section{Methods}

\subsection{HCN4 channel conductance}

The conductance of the funny channel $\left(g_{f}\right)$ in WT and WT0.5+DM conditions was estimated as the slope of the regression line of the $\mathrm{I} / \mathrm{V}$ graphs at hyperpolarized voltages (between -160 and $-115 \mathrm{mV}$ ), from Servatius et al. (See Figure 2, panel C [3]) and subtracting the slope of the I/V of the cells transfected with GFP (Green Fluorescent Protein) in order to remove non-specific leak:

scaling factor $=\frac{\text { slope }(W T 0.5+D M)-\operatorname{slope}(G F P)}{\operatorname{slope}(W T)-\operatorname{slope}(G F P)}$

The loss of function due to the WT0.5+DM was included in the single cell SAN model downscaling the nominal $g_{f}$ according to this scaling factor. 


\subsection{Cellular Coupling}

Starting from the single cell human SAN model by Fabbri et al. [4], the 1D and 2D models were obtained by modifying the membrane voltage $V_{m}$ update equation, adding the gap junction current term:

$$
\frac{\delta V_{m}}{\delta t}=\frac{-i_{\text {tot }}}{C_{m}}+\frac{V_{\text {net }}}{\rho_{\text {gap }} \cdot C_{m}}
$$

where $i_{t o t}$ is the sum of all the intracellular ionic currents present in the single cell model, $C_{m}$ is the membrane capacitance $(57 \mathrm{pF}), \rho_{\text {gap }}$ is the gap junction resistivity and $V_{\text {net }}$ is the voltage difference between 2 (in 1D) or 4 (in 2D) neighbouring cells. Different values of $\rho$ were used: $10,100,1000,10000$ and $\infty \mathrm{M} \Omega \cdot \mathrm{m}$ in order to test the coupling strength.

\subsection{Cellular Heterogeneity}

The $C a^{2+}$ permeabilities $\left(P_{C a L}, P_{C a T}\right)$, the maximal conductances $\left(g_{K r}, g_{K s}, g_{K u r}, g_{N a}, g_{f}, g_{t o}\right)$, the maximal activity of $\mathrm{Na}^{+} / \mathrm{K}^{+}$pump and the $\mathrm{Na}^{+} / \mathrm{Ca}^{2+}$ exchanger $\left(I_{N a K \max }, K_{\mathrm{NaCa}}\right)$ of the Fabbri model were randomized to take into account the biological variability. A log-normal distribution was used to avoid negative values, and the randomization was executed at different levels of $\sigma: 0.05,0.1,0.1873,0.3$ and 0.4 .

\subsection{Features extraction}

CL was computed as the difference in time between the last two Overshoots (OS) of the AP of every cell, since at the end of the $20 \mathrm{~s}$ the cells were supposed to be in steadystate. CV describes how fast the AP propagates through the tissue. To estimate the $\mathrm{CV}$, we measured the difference in time between the occurrence of the zero-crossing of the AP of the earliest activation cell and that of a cell distant 20 position from it. The cells were supposed to be $50 \mu \mathrm{m}$ long (central SAN cell) and CV constant.

\section{Results}

\subsection{Single Cell}

The heterozygous mutation brings to a loss of function of the HCN4 channel: the conductance $g_{f}$ drops to the $45 \%$ of the WT condition (Table 1). As predictable by this result, the CL of the WT0.5+DM case is longer than the WT one: $924 \mathrm{~ms}$ with respect to $814 \mathrm{~ms}(+13.5 \%)$. This is reflected in the substantial decrease in the Heart Rate (HR), which drops from $74 \mathrm{bpm}$ to $65 \mathrm{bpm}(-12.2 \%)$. Therefore, at the single cell level, a bradycardic effect is obtained due to the presence of the mutations.
Table 1. Percentage values of maximum $I_{f}$ conductance with respect to the wild type condition $\left(g f_{\max } / g f_{\max }(W T)\right)$ and correspondent absolute $g_{f}$ values; Cycle length (CL, in ms); Heart rate (HR, in beats per minute) and HR percentage variation.

\begin{tabular}{lrcr}
\hline \hline & & WT & WT0.5+DM \\
\hline $\mathbf{g f}_{\max } / \mathbf{g f}_{\max }(\mathbf{W T})$ & $100 \%$ & $45 \%$ \\
$\mathbf{g}_{\mathbf{f}}$ & {$[\mathrm{nS}]$} & 4.27 & 1.92 \\
$\mathbf{C L}$ & {$[\mathrm{ms}]$} & 814 & 924 \\
$\mathbf{H R}$ & {$[b p m]$} & 74 & 65 \\
$\Delta \mathbf{H R}$ & & - & $-12.2 \%$ \\
\hline \hline
\end{tabular}

\subsection{Dependence of CL with respect to $\rho$}

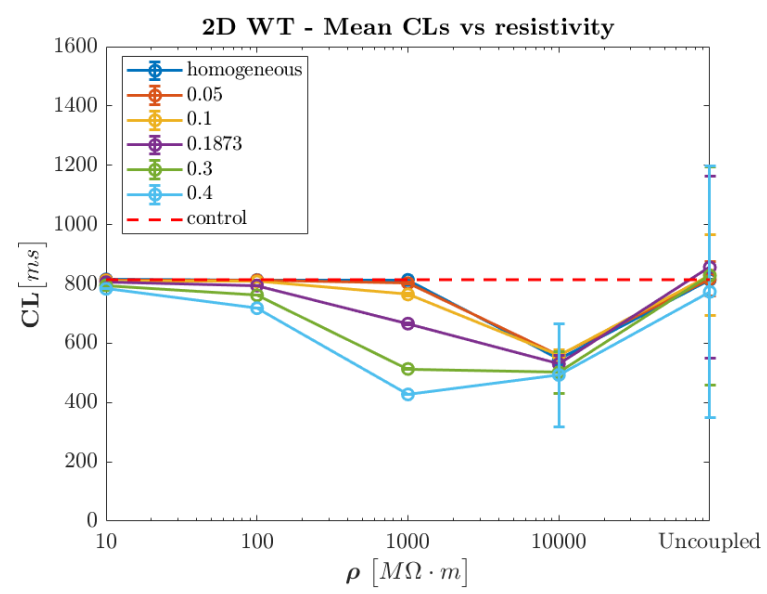

Figure 1. Trends of CL ( \pm std) with respect to resistivity for different levels of cellular heterogeneity and nominal value $(\mathrm{CL}=814 \mathrm{~ms}$, dashed line); 2D WT condition.

Figure 1 reports the mean CL at different degrees of coupling for the 2D tissue. The increase in gap junction resistivity provides a shortening in the mean CLs until a threshold value $(1000 / 10000 \mathrm{M} \Omega \cdot \mathrm{m}$ depending on $\sigma)$ over which CL starts to increase. Furthermore, under this value the cells are perfectly synchronized in terms of frequency independently from $\sigma$.

The trends for the WT0.5+DM condition are similar (Figure 2); in this scenario, most of the mean CLs have values higher than $814 \mathrm{~ms}$ (i.e. the duration of the single cell CL). This is due to the reduction of available $I_{f}$ current, in agreement with the single cell case.

\subsection{Dependence of $\mathrm{CL}$ with respect to $\sigma$}

The increase in heterogeneity of cellular parameters provides a decrease in the mean CLs for each value of $\sigma$, contrarily to what happened with $\rho$ (Figure 3). Moreover, this 


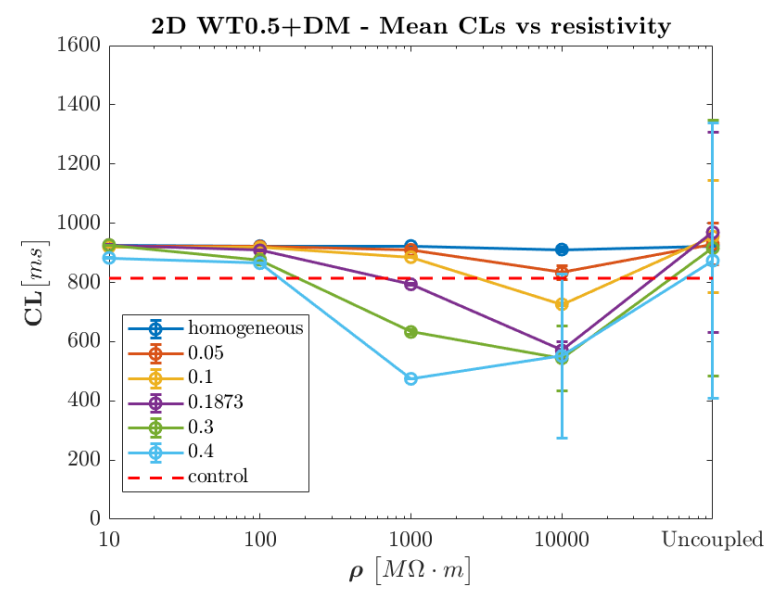

Figure 2. Trends of CL ( \pm std) with respect to resistivity for different levels of cellular heterogeneity and nominal value $(\mathrm{CL}=814 \mathrm{~ms}$, dashed line); 2D WT0.5+DM condition.

increase in the mean HR is consistent with the increase in dispersion: the higher the $\sigma$ the more difficult it is for the cells to pace at the same frequency. This is particularly true for poor cellular coupling as $\rho=10000 \mathrm{M} \Omega \cdot \mathrm{m}$ and $\infty \mathrm{M} \Omega \cdot \mathrm{m}$ (i.e. uncoupled cells), since these conditions prevent cells from interacting with one another.

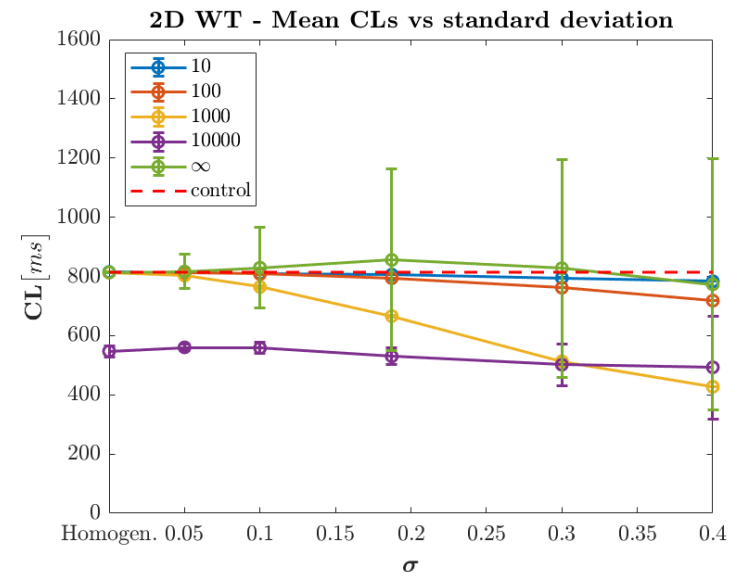

Figure 3. Trends of CL ( \pm std) with respect to cellular heterogeneity for different levels of cellular coupling and nominal value $(\mathrm{CL}=814 \mathrm{~ms}$, dashed line); 2D WT condition.

In the WT0.5+DM condition, the 2D tissue (Figure 4) showed similar trends to WT: the mutations only affect the mean CLs values - and not the dependence from $\sigma$.

Interestingly, in both WT and WT0.5+DM condition, for $\rho=10000 \mathrm{M} \Omega \cdot \mathrm{m}$ low CLs are obtained even with small $\sigma$, but they do not decrease much even with large standard deviations. In particular, for the WT homoge- neous condition, with $\rho=10000 \mathrm{M} \Omega \cdot \mathrm{m} \mathrm{CL}$ is not equal to the nominal CL value (Figure 3 ) because in that value synchronization (in phase) is not reached, and many leading cells drive the tissue, thus raising the frequency.

About the 1D model, CLs are shorter with respect to the 2D condition: with $\sigma=0.4$ and $\rho=1000 \mathrm{M} \Omega \cdot \mathrm{m}$ for example, $\mathrm{CL}=370 \mathrm{~ms}$ for the WT condition and 455 $\mathrm{ms}$ for the WT0.5+DM one. With more realistic values of the parameters $(\sigma=0.1873$ and $\rho=100 \mathrm{M} \Omega \cdot \mathrm{m})$ the obtained CLs are still shorter: $681 \mathrm{~ms}$ (WT) and $827 \mathrm{~ms}$ (WT0.5+DM). With respect to the 2D results - with the same values of the parameters - this means a difference of the $-14.2 \%$ for the WT condition and of the $-8.9 \%$ for the WT0.5+DM one.

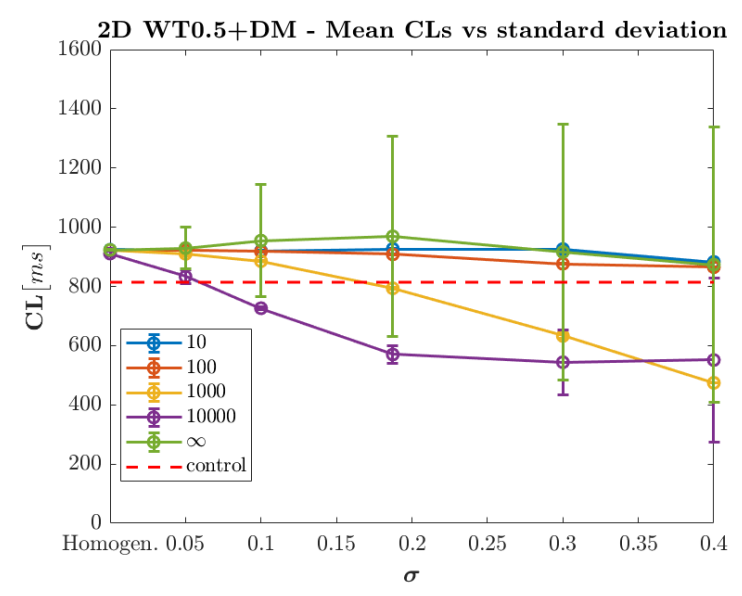

Figure 4. Trends of CL ( \pm std) with respect to cellular heterogeneity for different levels of cellular coupling and nominal value $(\mathrm{CL}=814 \mathrm{~ms}$, dashed line); 2D WT0.5+DM condition.

\subsection{Conduction velocity}

Table 2. Results for Conduction Velocity (in $\mathrm{cm} / \mathrm{s}$ ). In all the simulations $\sigma=0.1873$ was set, whereas $\rho$ was $50 \mathrm{M} \Omega \cdot \mathrm{m}$ in $1 \mathrm{D}$ and $100 \mathrm{M} \Omega \cdot \mathrm{m}$ in $2 \mathrm{D}$, in which case velocity was computed on both $\mathrm{x}$ and $\mathrm{y}$ axes. In $1 \mathrm{D}$, values are reported as mean \pm std $(n=5)$.

\begin{tabular}{lccccr}
\hline \hline & & \multicolumn{2}{c}{ 1D } & \multicolumn{2}{c}{ 2D } \\
\hline & & WT & WT0.5 & WT & WT0.5 \\
& & & + DM & & + DM \\
$\mathbf{C V}[\mathbf{c m} / \mathbf{s}]$ & $\mathbf{x}$ & $13 \pm 1.2$ & $13.1 \pm 1.6$ & 10.8 & - \\
& $\mathbf{y}$ & & & 12.1 & 10.6 \\
\hline \hline
\end{tabular}

Table 2 reports the estimated CV in 1D and 2D tissue. We identified $\rho$ and $\sigma$ on the basis of CV reported in literature [5]. We adopted $\sigma$ corresponding to physiological het- 
erogeneity ( $\sigma=0.1873$ ) in both $1 \mathrm{D}$ and 2D models. We observed physiological values of $\mathrm{CV}$ for $\rho=50 \mathrm{M} \Omega \cdot \mathrm{m}$ and $100 \mathrm{M} \Omega \cdot \mathrm{m}$ in $1 \mathrm{D}$ and $2 \mathrm{D}$ respectively.

In $1 \mathrm{D}$, a paired t-test was executed on $\mathrm{n}=5 \mathrm{CV}$ values extracted from simulations with different initial conditions and heterogeneity to check if the mutations affected the CV. Being the answer negative $(p=0.86)$, it seems that the DM only affects the HR: in 1D the obtained CLs are $690 \mathrm{~ms}$ and $802 \mathrm{~ms}$ for WT and WT0.5+DM respectively, whereas in 2D CL is $794 \mathrm{~ms}$ for WT and $908 \mathrm{~ms}$ for WT0.5+DM. In terms of frequency, this means $87 \mathrm{bpm}$ in WT and $75 \mathrm{bpm}$ in WT0.5+DM $(\Delta H R=-13.8 \%)$ in $1 \mathrm{D}, 76 \mathrm{bpm}(\mathrm{WT})$ and $66 \mathrm{bpm}(\mathrm{WT} 0.5+\mathrm{DM})$ in $2 \mathrm{D}$ $(\Delta H R=-13.2 \%)$.

\section{Discussion and Conclusion}

This work presents the investigation of the effects of the I479V/A485E double mutation [3] on human-specific models of the sinoatrial node. For the single cell simulations, the Fabbri model [4] was used; the 1D and 2D models were derived by combining together many of these single cell models.

About the mutations, their effect is represented only by a slow down in pacing frequency: at the single cell level the HR passes from $74 \mathrm{bpm}$ of the WT condition to the $65 \mathrm{bpm}$ in presence of the DM (-12.2\%). This is because of the drop in the conductance of the funny current $\left(g_{f}(W T 0.5+D M)=45 \%\right.$ of $\left.g_{f}(W T)\right)$ which stretches the diastolic depolarization phase, thus determining a longer CL. This is true also in 2D, where HR passes from $76 \mathrm{bpm}(\mathrm{WT})$ to $66(\mathrm{DM})$ when $\sigma=0.1873$ and $\rho=100 \mathrm{M} \Omega \cdot \mathrm{m}$. This means that, with these supposed physiologic levels of cellular heterogeneity and coupling, a bradycardic effect can be seen to affect the SAN. The 1D model is no exception (HR drops from $87 \mathrm{bpm}$ to $75 \mathrm{bpm}$, with $\sigma=0.1873$ and $\rho=50 \mathrm{M} \Omega \cdot \mathrm{m}$ ), but this case is less reliable since the approximation is substantial. In particular, in a fibre every cell only sees 2 connections with other cells: this represents a condition in which the resistance between cells is higher than when the connections are 4 (as in the 2D model). In the latter condition indeed, cells have an additional "node" in common and this makes cells to be partially in parallel, thus contributing to lower the actual resistance seen by them. As a consequence, the fast-pacing cells undergo a bigger influence from their neighbours, that in this way contribute to inhibit them by prolonging the diastolic depolarization phase. As a result hence, the frequency is lower. The difference between 1D and 2D models suggest how a more accurate description of the geometry (for example considering 3 dimensions) could provide different values of $\sigma$ and $\rho$ (in particular minor than 0.1873 and $100 \mathrm{M} \Omega \cdot \mathrm{m}$ ), since the inclusion of 2 more connections would further lower the resistance. As a result, the HR would be even closer to the $74 \mathrm{bpm}$ of the single cell reference value. This is of course to be confirmed by experimental data, but these models can provide also more information, such as the effect of pathological conditions as fibrosis, where $\rho$ is incremented: the simulations show how a bigger $\rho$ tends to shift the HR to higher values. Thus, these results could let think of a tachycardic effect of fibrosis.

This work aimed at providing a mechanistic interpretation of the clinic phenotype observed on a patient carrying the I479V/A485E double mutation; for this reason a simple geometry and constant values of $\rho$ were used, so as to isolate possible confounding effects. In order to further mimic the physiology of the SAN however, it would be appropriate to extend the geometry to the $3 \mathrm{D}$ case and to include a spatial gradient in cellular coupling [6], aspects that will be hopefully taken into account in future works.

\section{Acknowledgments}

$\mathrm{AF}$ and TdB acknowledge support from the ZonMW MKMD programme [grant number 114022502]. The authors thank Dr. A. Porro for providing raw data of the DM.

\section{References}

[1] Verkerk AO, Wilders R. Pacemaker activity of the human sinoatrial node: an update on the effects of mutations in HCN4 on the hyperpolarization-activated current. International Journal of Molecular Sciences 2015;16.

[2] Baruscotti M, Bucchi A, et al. A gain-of-function mutation in the cardiac pacemaker HCN4 channel increasing cAMP sensitivity is associated with familial Inappropriate Sinus Tachycardia. European Heart Journal 2017;38.

[3] Servatius H, Porro A, et al. Phenotypic spectrum of HCN4 mutations. Circulation Genomic and Precision Medicine 2018;11.

[4] Fabbri A, Fantini M, Wilders R, Severi S. Computational analysis of the human sinus node action potential: model development and effects of mutations. The Journal of Physiology 2017;595.

[5] Li N, Kalyanasundaram A, et al. Impaired neuronal sodium channels cause intranodal conduction failure and reentrant arrhythmias in human sinoatrial node. Nature Communications 2020;11.

[6] Inada $\mathrm{S}$, Zhang $\mathrm{H}$, et al. Importance of gradients in membrane properties and electrical coupling in sinoatrial node pacing. PLOS ONE 2014;9.

Address for correspondence:

Stefano Severi

Department of Electrical, Electronic and Information Engineering, University of Bologna,

Via dell'Università 50, 47522 Cesena (FC), Italy

stefano.severi@unibo.it 